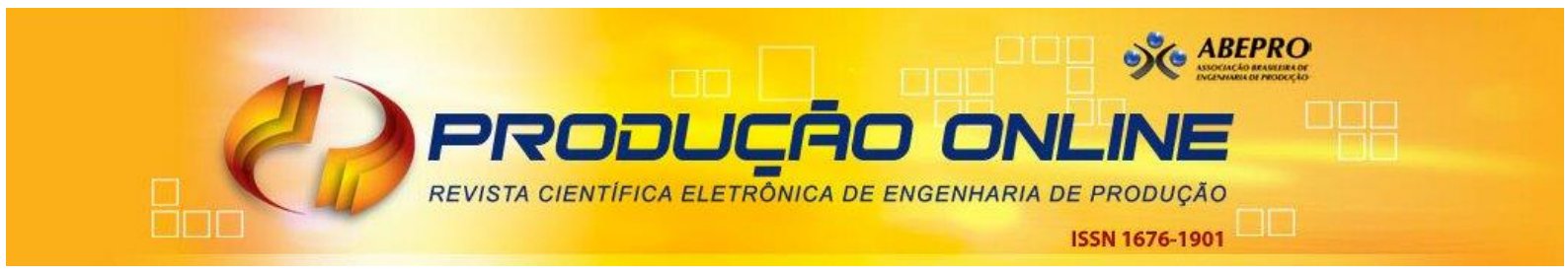

\title{
ANÁLISE COMPARATIVA DE MÉTODOS PARA CONFIABILIDADE HUMANA
}

\section{COMPARATIVE ANALYSIS OF HUMAN RELIABILITY METHODS}

\author{
Stela Xavier Terra* E-mail: stela.xavier.terra@gmail.com \\ Ariane Ferreira Porto Rosa* E-mail: afprosa61@gmail.com \\ Jaime de Paula Neto* E-mail: jaime.paula.neto@gmail.com \\ Witielo Arthur Seckler* E-mail: witieloseckler@gmail.com \\ *Universidade Federal de Pelotas (UFPel), Pelotas, RS
}

Resumo: $O$ não ajuste das condições de trabalho podem gerar acidentes, incidentes e até doenças laborais que levam ao afastamento do trabalho, podendo ser permanente. Tal situação reduz os recursos humanos disponíveis e sobrecarrega os seus pares repercutindo num efeito looping. $\mathrm{O}$ uso inadequado da tecnologia pode comprometer o desempenho operacional de aparelhos, máquinas e equipamentos. Muitas vezes o mau uso pelo operador do ativo físico pode gerar consequências sérias como, por exemplo, o acidente de Three Mile Island (1979). Ressalta-se assim a importância do fator humano na confiabilidade de um sistema. A confiabilidade humana como ciência é aplicável à vários setores da indústria e tem por propósito analisar o erro humano e seu impacto na produtividade, segurança e qualidade. Tendo o fator humano como um elo crítico de um sistema, o principal objetivo do presente artigo é comparar a utilidade de determinados métodos de avaliação de confiabilidade humana. Evidenciando-se as semelhanças e, principalmente, as diferenças entre os métodos abordados, sendo eles: THERP, SLIM-MAUD, ATHEANA, CREAM e MERMOS. Assim, foi desenvolvida uma pesquisa exploratória sustentada por estudo bibliográfico, além de um quadro comparativo destes métodos. Com este estudo constatou-se que os procedimentos de predição e análise de falhas, até então voltados para projetos e processos industriais, associados a falhas de equipamentos, podem ser ampliados para utilização no controle e prevenção das falhas cometidas por pessoas.

Palavras-Chave: Confiabilidade Humana. Erro Humano. Avaliação da Confiabilidade Humana.

\begin{abstract}
Failure to adjust working conditions can lead to accidents, incidents, and even occupational diseases that lead to work withdrawal and may be permanent. Such a situation reduces the available human resources and overwhelms their peers, resulting in a looping effect. Inappropriate use of the technology may compromise the operational performance of appliances, machinery and equipment. Often the misuse by the physical asset operator can have serious consequences, such as the Three Mile Island accident (1979). Thus, the importance of the human factor in the reliability of a system is emphasized. Human reliability as a science is applicable to various industry sectors and its purpose is to analyze human error and its impact on productivity, safety and quality. Taking the human factor as a critical link of a system, the main objective of this paper is to compare the usability of certain human reliability assessment methods. The similarities and, mainly, the differences between them are shown, being the techniques choosen THERP, SLIM-MAUD, ATHEANA, CREAM and MERMOS. Thus, an exploratory research supported by a bibliographic study was developed, as well as a comparative table of these methods. With this study it was verified that the procedures of prediction and analysis of failures, hitherto focused on industrial projects and processes, associated to equipment failures, can be extended for use in the control and prevention of failures committed by people.
\end{abstract}

Keywords: Human Reliability. Human Error. Human Reliability Assessment. 


\section{INTRODUÇÃO}

Um ritmo muito rápido de tecnologia é observado no nível operativo da sociedade em muitos domínios, como aeronáutica, setor de energia elétrica, indústria de processo, manufatura, transporte, embarque, organizações essencialmente apoiadas em sistemas de TI (bancos internacionais), entre outros. No entanto, as estruturas organizacionais e de gestão nesses domínios são bastante conservadoras, em vários sentidos, entre eles está a ideia de que erros humanos são frequentemente associados a trabalho de má qualidade, falhas e fraquezas pessoais. É por isso que, acredita-se ser desagradável discuti-los abertamente em meios laborais. No entanto, os erros não são necessariamente um resultado de descuido ou falta de capacidade ou habilidade. Uma pessoa pode estar distraída, cansada ou sobrecarregada. Questões pessoais não são as únicas razões possíveis para isso. $O$ problema pode, de fato, estar no ambiente de trabalho.

Atualmente grandes desenvolvimentos tecnológicos, sobretudo na biologia e medicina, pressionam os seres humanos por maior capacitação física e mental atendendo à sociedade altamente competitiva. O não ajuste das condições de trabalho podem gerar acidentes, incidentes e até doenças laborais que levam ao afastamento do trabalho, podendo ser permanente. Tal situação reduz os recursos humanos disponíveis e sobrecarrega os seus pares repercutindo num efeito looping.

O uso inadequado da tecnologia pode comprometer 0 desempenho operacional de aparelhos, máquinas e equipamentos. Muitas vezes o mau uso pelo operador do ativo físico pode gerar consequências sérias. Como as que acontecem em acidentes aéreos, usinas nucleares, plataformas offshore. Nesse contexto, o desempenho do sistema homem-máquina é crítico, como por exemplo, o acidente de Three Mile Island (TMI) em 1979, cujo papel crítico dos operadores humanos em garantir a segurança das instalações tornou-se o fator desencadeante de pesquisas, principalmente, no setor de energia nuclear.

O acidente da TMI foi um alerta para a indústria nuclear em todo o mundo. Apontou a importância do operador como barreira de segurança e a necessidade de ferramentas de treinamento e suporte de alta qualidade para obter alto desempenho humano em situações complexas. TMI tem sido de interesse para engenheiros de 
fatores humanos por ser um exemplo de como grupos de pessoas reagem e tomam decisões sob estresse. Há consenso de que o acidente foi exacerbado por decisões erradas tomadas porque os operadores estavam sobrecarregados com informações, muitas delas irrelevantes, enganosas ou incorretas (ØWRE, 2011).

Os fatores elencados acima respaldam a importância do fator humano na confiabilidade de um sistema, a qual conceitua-se como a probabilidade de seus componentes cumprirem suas respectivas missões, isto é, a fiabilidade do sistema executar sua função durante um determinado tempo em condições previstas (LAFRAIA, 2001).

Nesse sentido, Komninakis, Piratelli e Achcar (2018), corroboram com a ideia de que Confiabilidade, mesmo que no sentido amplo, é um dos fatores cruciais para o sucesso das organizações. Sendo o fator humano um componente crítico em um sistema, o principal objetivo do presente artigo é traçar um comparativo, no qual visa-se compreender a utilidade de determinados métodos de avaliação de confiabilidade humana. Através de uma revisão bibliográfica buscou-se evidenciar as semelhanças e, principalmente, as diferenças entre os métodos mais abordados: THERP, SLIM-MAUD, ATHEANA, CREAM e MERMOS.

\section{MATERIAIS E MÉTODOS}

Modelos ou técnicas de avaliação da confiabilidade humana estão disponíveis na literatura, cada um com suas próprias características. No entanto, foram escolhidos pelos autores os 5 métodos mais abordados na literatura atual.

Em relação a classificação dos procedimentos metodológicos realizados, pode-se definir como uma pesquisa de natureza básica, a qual visa agregar conhecimentos úteis para o meio acadêmico-científico. Quanto ao procedimento técnico foi escolhida a pesquisa bibliográfica.

Por se tratar de uma revisão bibliográfica, na qual foram pesquisados estudos desenvolvidos por diversos autores a partir de casos específicos, pode-se classificar esta pesquisa quanto ao método científico de indutivo. Isto significa, que argumentos passam do particular para o geral, uma vez que as generalizações derivam de observações de casos da realidade concreta relatados nos artigos estudados. 
Para tanto foram consultados o Portal Periódicos e a ferramenta CAFe da CAPES, os quais tornaram esse trabalho possível, visto que estes permitem acesso a uma ampla variedade de bibliotecas digitais, bem como revistas. Somado a isto, utilizaram-se materiais literários da biblioteca eletrônica Scielo, e publicações de editoras renomadas como Elsevier, John Wiley \& Sons, Springer, entre outras. Dentre as palavras chave pesquisadas estão confiabilidade humana, técnicas de confiabilidade humana, e os nomes das técnicas estudadas: THERP, SLIM-MAUD, ATHEANA, CREAM e MERMOS.

No tocante aos objetivos da pesquisa, ela é caracterizada como exploratória, visando obter maior familiaridade sobre o assunto. Na fase preliminar, a pesquisa tem como propósito proporcionar mais informações sobre o objeto de estudo para possibilitar a formulação das hipóteses ou descobrir um novo tipo de enfoque para o assunto.

A abordagem da pesquisa é qualitativa. A interpretação dos fenômenos e a atribuição de significados são as premissas do processo de pesquisa qualitativa (PRODANOV \& FREITAS, 2013).

\section{CONFIABILIDADE HUMANA}

De acordo com Pallerosi et al. (2011) a confiabilidade humana (CH) pode ser definida como a qualidade ou estado de quem se pode confiar, ou seja, é a probabilidade de alguém ter sucesso no cumprimento da sua missão, em determinado período e condições ambientais apropriadas com recursos necessários disponíveis.

Nascimento Neto (2014) afirma que a CH como ciência é aplicável à vários setores da indústria e tem por propósito analisar o erro humano e seu impacto na produtividade, segurança e qualidade. Nesse contexto, o mesmo autor destaca a importância de elaborar estratégias para prevenir, mitigar ou eliminar erros para diminuir a frequência dos acidentes. Os acidentes decorrentes do erro humano podem tanto afetar o capital humano quanto causar ineficiência nos processos de uma indústria.

Diante dessas definições é possível inferir que a confiabilidade humana está associada à atividades laborais sendo a probabilidade de que uma tarefa atribuída, Revista Produção Online. Florianópolis, SC, v. 20, n. 4, p. 1170-1189, 2020. 
seja executada por uma pessoa conforme procedimentos específicos em condições pré-determinadas. Não executar essa função atribuída conforme regras e/ou procedimentos pode ser definido como uma erro humano que pode ou não ter consequências. $\mathrm{O}$ interesse no estudo da $\mathrm{CH}$ está principalmente ligado ao fato de que as consequências da falha humana podem levar a perdas material, ambiental e humana, podendo gerar um potencial catastrófico. Na literatura é usualmente mais abordado termos como erro humano em vez de falha humana sendo que as vezes seus termos são confundidos pelos autores que estabelecem o mesmo significado para ambos os termos.

Esses termos são confundidos nomeadamente pela atribuição de significado similar, por exemplo, reportar "falha humana" como simplesmente uma falha na ação humana, o que é na verdade um tipo de erro ainda não especificado, uma vez que não são caracterizadas decorrências (um evento) que interfiram na operação do equipamento ou sistema, portanto não é uma falha humana (SPURGIN, 2010).

A Sociedade Norte-americana de Engenheiros Mecânicos (2002), American Society of Mechanical Engineers (ASME) apud Gertman et al. (2005) define evento de falha humana como "evento que representa uma falha ou indisponibilidade de um componente, função ou sistema causada por inação humana ou uma ação inadequada". Ou seja, falha humana é produto de no mínimo um ato inseguro que inviabilizou um aspecto.

Uma estrutura de classificação de falhas razoável é apresentada na Figura 1, elabobrada pelo Health and Safety Executive (Executivo de saúde e segurança), que é o órgão responsável pelo incentivo, regulamentação e aplicação da saúde, segurança e bem-estar no local de trabalho e pela pesquisa sobre riscos ocupacionais na Grã-Bretanha e tem sua atuação datada desde o ano de 1974. 
Figura 1 - Fluxograma de classificação da falha humana

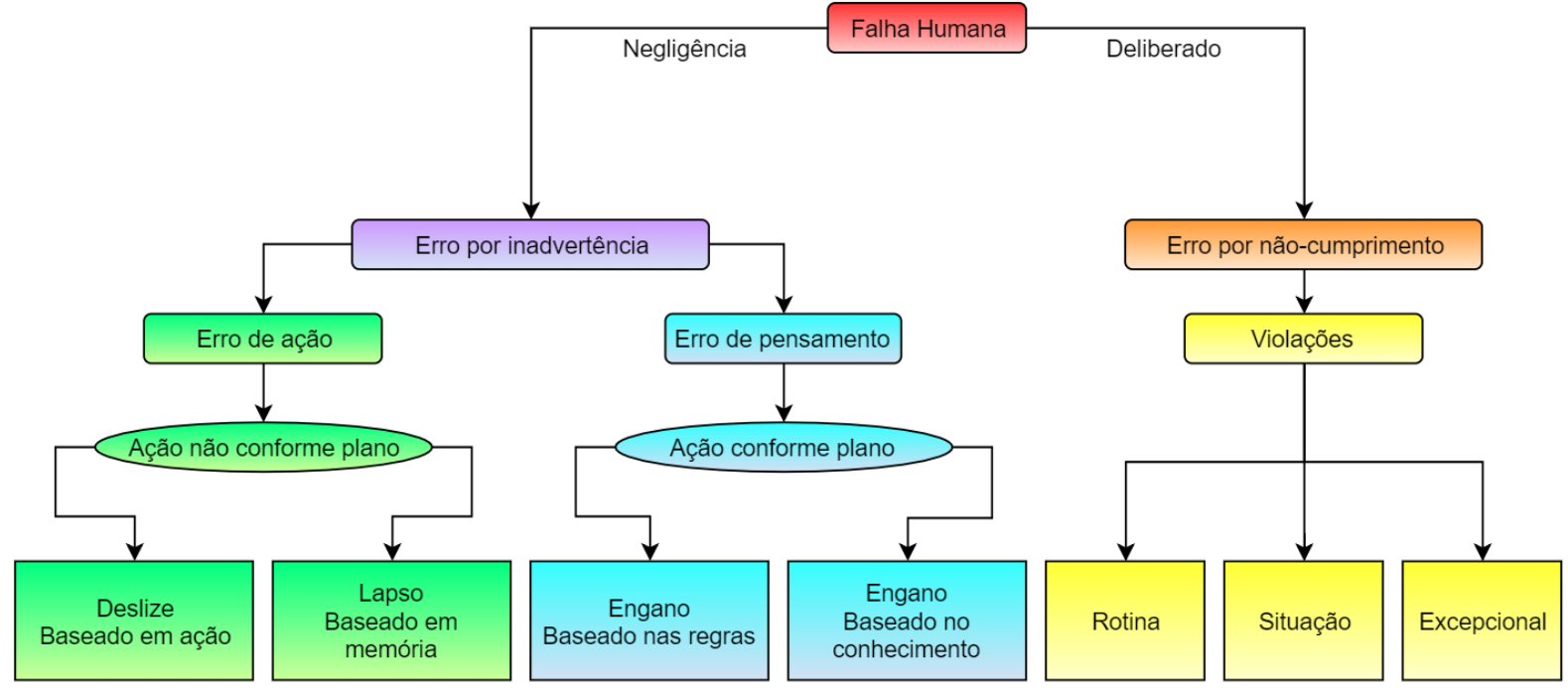

Fonte: Adaptado de Health and Safety Executive (2019)

\subsection{Interpretando erro por não-cumprimento}

Violações são os desvios deliberados das regras, procedimentos, regulamentos. Também conhecido por transgressões humanas ou não cumprimento. É caracterizado por ser algo feito conscientemente pelo executor, pegando atalhos ou não seguindo procedimentos, com algum intuito, por exemplo, para economizar tempo ou esforço (REASON, 1995). Geralmente pode ser bem-intencionado, porém equivocado já que a tarefa bem projetada geralmente considera as condições favoráveis de produtividade, segurança do trabalhador e condições ambientais, logo, uma execução que não siga os protocolos estabelecidos conscientemente pode resultar em falha.

Reason (1995) ainda os classifica em três tipos: i) Violação de rotina: em que o não cumprimento se torna recorrente; ii) Violação situacional: não conformidade ditada por fatores específicos da situação como pressão de tempo, carga de trabalho, ferramentas e equipamentos inadequados, clima etc. e; iii) Violação excepcional: quando tenta-se resolver uma situação altamente incomum, geralmente quando algo não resulta conforme esperado e então a pessoa assume o risco em transgredir o devido procedimento. 


\subsection{Interpretando erro por inadvertência}

Segundo a classificação extraída da Health and Safety Executive (2019) cujo o fluxograma é ilustrado na Figura 1, seguem as duas classificações para erro por inadvertência:

i) Erro de ação: que é quando a ação ocorre de forma não planejada, ou seja, em que a ação resultante não é pretendida, geralmente associado a tarefas familiares que exigem pouca atenção consciente. Esses erros baseados em habilidades ocorrem quando a atenção é desviada, mesmo que momentaneamente. Ainda se subdivide em deslizes: uma ação física simples que executada com frequência e dá errado como por exemplo quando um auxiliar de cozinha se fere cortando o alimento repetidas vezes em tamanhos similares; e lapso: lapso na memória de curto prazo ou omissão para executar uma ação necessária, como esquecer de dar seta no trânsito por exemplo;

ii) Erro de pensamento: podem ser do tipo erro baseado em regras, quando são falhas de decisão ou erros de julgamento, ou ainda erro baseado no conhecimento, que é caracterizado pela ação realizada conforme planejado, usando processos de pensamento conscientes, mas o curso errado da ação é tomado, ou seja, quando a pessoa faz algo errado pensando estar fazendo o certo.

\subsection{Compreendendo o conceito de erro humano}

Rigby (1970) define o Erro Humano (EH) como qualquer elemento pertencente ao conjunto de ações humanas que excede algum limite de aceitabilidade. Para Lafraia (2001) é definido como "a falha de ações planejadas em alcançar os objetivos propostos". Dessa forma, o EH tem duas causas: (i) ações não ocorrem como planejadas e (ii) o planejamento foi inadequado.

Dekker (2006) destaca o termo "Erro Humano" como sendo frequentemente considerado pejorativo, o que sugere que a causa do modo de falha é da natureza do ser humano. Boring (2014) aborda a corrente mais aceita, na qual entende-se erro humano como um produto do contexto em que o ser humano opera. Em outras palavras, a causa final do erro não é o humano em si, mas sim os mecanismos que 
podem proporcionar uma situação geradora da falha, como fatores ambientais ou o projeto do processo em sí, por exemplo que o colocariam em uma situação tal qual fosse provável que o erro ocorresse.

Neste contexto, erros e problemas comportamentais reduzem a produtividade, a qualidade e a segurança dos processos sociais, industriais e de qualquer outra atividade humana gerando um custo social elevado. Esse custo social, se levado à um nível aceitável, poderia liberar recursos essenciais para contribuir para o desenvolvimento do coletivo.

A avaliação da confiabilidade humana, em inglês Human Reliability Assessment (HRA), utiliza métodos que, geralmente, identificam um conjunto de fatores que se acredita estar relacionado ao desempenho, enfocando classes de erro ou comportamento humano ao manipular esses fatores a fim de chegar à estimativa de taxa de falha para cada um. Partindo dessa premissa, é selecionada uma ou mais técnicas de HRA conforme os aspectos do sistema a ser avaliado, e após aplica-se suas respectivas metodologias. Entre alguns métodos existem a THERP, o SLIM-MAUD, o CREAM e o MERMOS, apresentados a seguir.

\subsection{Método THERP}

O método denominado Taxa de Predição para Taxa do Erro Humano, em inglês Technique for Human Error Rate Prediction (THERP) foi desenvolvido para fornecer modelagem representacional de ações humanas (árvores de evento de HRA) e estimativa da probabilidade de erro humano, HEP, do inglês Human Error Probability (GERTMAN et al., 2005). Há muitas maneiras de estimar as HEPs, algumas são estatísticas e outras vêm do julgamento de especialistas. O Manual do THERP usa o conceito HEP para representar qualquer estimativa (ALVARENGA; FONSECA, 2009).

De acordo com Spurgin (2010) a ferramenta THERP é baseada nos resultados de uma análise de tarefas, que divide uma tarefa em várias subtarefas. No referido manual a realização de uma matriz gera uma montagem de subtarefas de HRA discretas, formando uma árvore de eventos de HRA. Para quantificar essa 
árvore de eventos, deve-se selecionar as HEPs apropriadas para corresponder às subtarefas.

A abordagem do manual está estruturada em um modelo do tipo ferramenta de fatores humanos, a análise de tarefas. No processo de análise de tarefas, qualquer tarefa é dividida em vários elementos ou subtarefas. Após, deve-se alocar um valor HEP para cada elemento ou subtarefa com base na identificação da subtarefa em uma tabela prévia de consulta, contendo preferencialmente descrição. Cada uma das subtarefas é então representada em uma árvore de eventos de confiabilidade humana. O total de HEPs na árvore é somado para fornecer uma HEP total.

THERP é o método de HRA mais documentado. Entretanto, o seu uso não é recomendado para avaliar processos cognitivos dependentes de tempo, como o diagnóstico de um monitor de sistema de alarmes durante um acidente. A modelagem de ações humanas utilizando árvores binárias de eventos apresenta-se difícil, pois dessa forma não considera a dependência entre eventos e entre fatores de desempenho. Como o comportamento humano é imprevisível, faz-se necessário identificar todas as possíveis ações erradas causadoras de um evento de falha. As árvores de eventos por apresentarem lógica binária, demandarão muitos ramos, tornando a análise complexa (KUMAMOTO; HENLEY, 1996).

\subsection{Método SLIM-MAUD}

Success Likelihood Index Method, ou Método do Índice de Probabilidade de Sucesso não é um método de HRA per se, mas sim uma técnica de escalonamento. Não possui um conjunto fixo de HEPs nem possui um conjunto necessário de fatores de modelagem de desempenho, Performance Shaping Factors (PSFs). O método usa a Multi-Attribute Utility Decomposition (MAUD), decomposição utilitários multiatributos e representa uma abordagem predominantemente psicológica em oposição às abordagens baseadas em engenharia e confiabilidade, como a THERP (CHANDLER et al., 2006).

Segundo Hollnagel (1998) SLIM-MAUD é uma abordagem para estimar as HEPs por meio de um procedimento interativo baseado em computador, o MAUD, 
que serve para elucidar e organizar as avaliações de especialistas dentro da estrutura do SLIM. Assim, essa abordagem baseia-se na suposição de que a probabilidade de falha associada ao desempenho da tarefa é derivada de uma combinação de PSFs que incluem as características do indivíduo, do ambiente e da tarefa. Uma segunda suposição é que os especialistas podem estimar essas taxas de falha ou selecionar valores de âncora razoáveis para permitir que o software calcule a estimativa.

Para Chandler et al. (2006) o SLIM-MAUD veio formalizar o uso de julgamento especializado na estimativa dos valores de HEP, requerendo observações (data points) mínimos como, por exemplo, estatísticas de eventos reais para avaliação da HEP. Embora o método tenha sido amplamente utilizado na avaliação probabilística de risco, Probabilistic Risk Assessment (PRA) nuclear, como uma estrutura computacional, ele pode ser facilmente aplicado a outros domínios.

\subsection{Método ATHEANA}

Segundo Serrano (2009) a Técnica para Análise de Eventos Humanos (TAEH), do inglês $A$ Technique for Human Event Analysis, é uma das mais utilizadas na indústria de produção de energia nuclear a nível mundial, devido a sua consideração acerca dos cenários de potenciais acidentes, incluindo os mais recentes avanços das ciências cognitivas.

Pinto (2014) afirma que TAEH se destaca por ser uma modelagem das interações entre o operador e o contexto onde ele está inserido. Tal afirmação corrobora com os autores Bye e Braarud (2004) de que a técnica é uma avaliação da situação, monitorando e detectando tanto a resposta do planejamento quanto a sua reposta de implementação.

O guia da ATHEANA desenvolvido por Forester et al. (2007) contempla uma lista de fatores como, por exemplo, nível de treinamento, acesso aos procedimentos, nível de estresse, fadiga, condições ambientais, tempo disponível para realização de tarefas, entre outros. A lista é baseada em anos de pesquisa na área de confiabilidade humana. Segundo Chandler et al. (2006) a técnica ATHEANA fornece uma boa base para discussões sobre o gerenciamento de instalações químicas. 
A técnica inicia com uma identificação de possíveis eventos de falha humana (HFE), associando o evento básico da APS à perda de função de um componente/sistema. Após, Ações Inseguras (AI), as chamadas Unsafe Acts (UA), são identificadas como os atos responsáveis pela ocorrência dos HFE. Em seguida, os contextos dessas ações são discriminados. Consideram-se para cada contexto diferente a combinação entre condições específicas do local e PSFs, isto é, fadiga, estresse, ruído, instrumentação, etc. Ao final, todas as considerações influenciarão na quantificação das probabilidades de ocorrência dos HFE. A quantificação é realizada através da opinião dos especialistas de acordo com os princípios supracitados (SILVA et al., 2017; PINTO, 2014).

\subsection{Método CREAM}

The Cognitive Reliability Error Analysis Method (CREAM) foi desenvolvido por Erik Hollnagel (1998) com base na experiência em ambos os campos de fatores humanos e HRA. Conforme Serrano (2009) o CREAM é uma metodologia ampla que pode atender as demandas por HRA de vários tipos de indústria. Já se tem notícia de indústrias químicas e em cenários de acidentes ferroviários. O primeiro passo é fazer uma análise de tarefas por meio de uma lista das atividades dos operadores. Para cada atividade é determinado o nível das Condições de Desempenho Comum (CDC). O próximo passo é descrever cada atividade cognitiva em termos de observação, interpretação, planificação e execução, assim como representar graficamente estas funções.

Depois de feita a classificação "fenótipo-genótipo", cria-se uma lista das falhas de funções cognitivas, subdividindo-as em vários sub-conjuntos. Assim, dentro de todo o subconjunto, cada função cognitiva (erros de observação, interpretação, planificação e execução), terá falhas potenciais identificadas. Para estas falhas é calculada a probabilidade baseada no fator das influências contextuais (CDCs), além de serem representadas graficamente. A metodologia CREAM descreve regras para quantificação dos CDCs (SERRANO, 2009).

A principal característica do método é que ele é totalmente bidirecional, ou seja, os mesmos princípios podem ser aplicados para análise retrospectiva (a busca 
de causas) e para previsão de desempenho. O modelo é baseado em uma distinção fundamental entre competência e controle, que oferece uma maneira de descrever como o desempenho depende do contexto. Finalmente, o esquema de classificação separa claramente as causas (genótipos) e manifestações (fenótipos) e, além disso, propõe uma organização não hierárquica de grupos de genótipos (HOLLNAGEL, 1998).

Segundo Hollnagel (1998) o esquema de classificação consiste basicamente em um número de grupos que descrevem os fenótipos (modos de erro, manifestações) e genótipos de ações errôneas, onde os últimos se referem a uma distinção fundamental entre genótipos de uma pessoa, tecnologia e organização relacionadas à natureza. Para cada grupo de classificação, é dada uma definição dos consequentes gerais e específicos que descrevem as consequências observáveis ou inferidas. Em seguida, é dada uma definição das ligações entre consequentes e antecedentes para cada grupo de classificação.

Ao contrário de um esquema de classificação hierárquica tradicional, esses links descrevem muitos caminhos potenciais através dos grupos de classificação. A realização de um caminho específico depende das condições sob as quais a ação ocorre, portanto, automaticamente, leva em conta a influência do contexto. Esse é o caso tanto da propagação reversa (análise de acidentes) quanto da propagação direta (previsão de desempenho). Em ambos os casos, a propagação é limitada por informações sobre as condições de desempenho e a profundidade da análise é determinada por regras de parada predefinidas (HOLLNAGEL, 1998).

\subsection{Método MERMOS}

É o atual método de HRA utilizado pela Electricité De France (EDF), companhia de eletricidade francesa. A EDF esteve envolvida em Análise Probabilística de Segurança (APS) por algum tempo. Eles publicaram seu primeiro relatório da APS sobre os Reatores de Água Pressurizada em 1985. A HRA foi baseada em uma simulação do tipo Curva de Confiabilidade do Tempo (CCT) por sessões no simulador em condições de acidentes. Como grupo, os investigadores 
franceses estavam mais interessados (dos efeitos de acidentes) nas respostas comportamentais das tripulações do que em suas ações no tempo.

Consequentemente, seu programa de simulador consistiu em grande número de diferentes cenários. Isso foi diferente do projeto Experiência de Confiabilidade do Operador (ECO) no instituto de pesquisa de energia elétrica, que focava em pequeno número de cenários e grande número de equipes. No projeto ECO, todas as equipes licenciadas pela Comissão Reguladora Nuclear de seis estações participaram dos estudos de acidentes simulados. A EDF abandonou a abordagem "antiga" da HRA e ao longo do tempo desenvolveu a abordagem MERMOS (SPURGIN, 2010).

A decomposição da sequência do acidente em várias ramificações é a task analysis e faz parte do método. Segundo Spurgin (2010) insights sobre o colapso são conduzidos pela experiência da EDF decorrente de seus anos de observação das sessões de simulação em vários simuladores para suas usinas de energia. Além disso, a experiência do simulador gera a possibilidade de dados reais, por causa do grande número de usinas da EDF quase, todas, idênticas. Assim as sessões podem ser usadas para treinar especialistas a fim de fazer estimativas mais informadas das probabilidades de erro humano de equipes. É difícil produzir uma descrição, passo a passo, da abordagem do MERMOS sem um profundo conhecimento do método e sua aplicação (por exemplo, quais dados reais estão disponíveis para uma determinada ação).

Portanto o Methode d'Evaluation de la Realisation des Missions Operateur la Sureté, isto é, o método para avaliação do desempenho da missão de segurança do operador diz respeito ao que é, de fato o sistema, qual é o evento inicial que causa um acidente e qual é a missão completa da equipe e do equipamento de segurança. A abordagem geral é a mesma que a maioria das PRAs, mas o pensamento sobre a combinação dos elementos é diferente. A equipe tem várias possibilidades para lidar com o impacto do evento inicial na fábrica, de modo que as respostas da equipe serão afetadas pela disponibilidade de segurança e outros equipamentos. Todos esses aspectos deverão ser levados em conta, e a história delineada (SPURGIN, 2010). 
Pesme, Le Bot e Meyer (2007) são enfáticos ao caracterizar o método como uma abordagem, entre aspas, bottom-up. Inicialmente, os autores revelam que os outputs da análise são o que eles chamam de cenários de MERMOS (MERMOS scenarios), sendo estes nada mais do que histórias curtas, das quais serão extraídas as hipóteses de como podem ocorrer as falhas. Isso permitirá uma primeira avaliação do risco, depois, um "olhar" mais próximo para cada output possibilitará a apreciação do seus componentes (e.g. fatores de risco e fatores que limitam a importância desses fatores de risco), e mostrar que o rigor de sua construção a ser verificado de um ponto de vista da $\mathrm{CH}$ significa tanto do ponto de vista da confiabilidade (abordagem sistêmica) como do ponto de vista da ciência comportamental, abordando a realidade do campo social (funcionamento em grupo).

\subsection{Quadro Comparativo}

O resultado da pesquisa bibliográfica do presente estudo está sumarizado no Quadro 1. No Quadro 1 a triagem refere-se à seleção preliminar e identificação de atividades humanas significativas para a PRA. A decomposição em tarefas identifica atividades humanas significativas de PRA em subtarefas para uma análise de erro mais específica. O modelo causal refere-se se uma cadeia causal é fornecida para o analista para identificar as "causas-raiz" das causas imediatas. Tais modelos causais podem ser usados em análise retrospectiva e identificação de causa raiz. A sua caracterização como "camada única" significa que apenas uma lista de PSFs é fornecida, enquanto a "multicamada" significa que uma cadeia causal explícita é fornecida, considerando interdependências de fatores causais. A cobertura inclui: ergonômica, cognitiva e organizacional. Este item observa se o método fornece instruções "relativamente detalhadas" para avaliar o efeito dos PSFs ou dos fatores no escopo específico do problema. Estimação de limites de incerteza refere-se se nenhum dos métodos que fornecem capacidade de ligação à incerteza faz uma distinção entre natureza aleatória ou epistêmica das incertezas). O termo "reprodutibilidade" refere-se ao nível de consistência nos resultados produzidos por indivíduos diferentes para a mesma tarefa. Enquanto "validação", refere-se à validação independente formal dos resultados. E "sensibilidade" trata-se da 
quantidade de alterações nos resultados numéricos quando os parâmetros de entrada são alterados.

Quadro 1 - Comparativo entre métodos de HRA

(continua)

\begin{tabular}{|c|c|c|c|c|c|}
\hline Técnica & Triagem & $\begin{array}{c}\text { Decomposição } \\
\text { em tarefas }\end{array}$ & \multicolumn{2}{|c|}{ Lista PSF } & Modelo causal \\
\hline THERP & Sim & $\begin{array}{l}\text { Triagem, } \\
\text { Diagnóstico e } \\
\text { Ação }\end{array}$ & \multicolumn{2}{|c|}{$\begin{array}{l}\text { 3, o THERP permite que o } \\
\text { usuário adicione PSFs } \\
\text { diferentes dos três PSFs } \\
\text { explicitamente especificados } \\
\text { (ou seja, Níveis de } \\
\text { Treinamento, Estresse e } \\
\text { Experiências) para ajustar os } \\
\text { valores de HEP, no entanto, } \\
\text { não fornece orientação sobre } \\
\text { o efeito desses PSFs para o } \\
\text { analista. }\end{array}$} & Única camada \\
\hline SLIM/MAUD & Não & $\begin{array}{c}\text { Não } \\
\text { especificado }\end{array}$ & \multicolumn{2}{|c|}{$\begin{array}{l}\text { Definido pelo usuário ( } 9 \\
\text { sugerido) }\end{array}$} & Única camada \\
\hline ATHEANA & Não & $\begin{array}{c}\text { Não } \\
\text { especificado }\end{array}$ & \multicolumn{2}{|c|}{ Definido pelo usuário } & $\begin{array}{c}\text { Implícito, o método } \\
\text { fornece instruções } \\
\text { "relativamente } \\
\text { detalhadas" para } \\
\text { avaliar o efeito das } \\
\text { PSFs ou dos fatores } \\
\text { no escopo } \\
\text { específico do } \\
\text { problema }\end{array}$ \\
\hline CREAM & Sim & $\begin{array}{l}15 \text { tipos de } \\
\text { tarefas }\end{array}$ & \multicolumn{2}{|c|}{$\begin{array}{c}9 \text { para quantificação; vários } \\
\text { para causas raiz }\end{array}$} & Multicamada \\
\hline MERMOS & Sim & $\begin{array}{l}\text { Triagem e } \\
\text { Diagnóstico }\end{array}$ & \multicolumn{2}{|c|}{ Definido pelo usuário } & Multicamada \\
\hline Técnica & \multicolumn{2}{|c|}{ Cobertura } & $\begin{array}{c}\text { Fonte primária } \\
\text { para } \\
\text { estimativas } \\
\text { HEP } \\
\end{array}$ & $\begin{array}{l}\text { Abordagem } \\
\text { da análise }\end{array}$ & $\begin{array}{l}\text { HEPs para modos } \\
\text { de erro específicos }\end{array}$ \\
\hline THERP & \multicolumn{2}{|c|}{ Ergonômica e organizacional } & $\begin{array}{l}\text { Números } \\
\text { fornecidos pelo } \\
\text { método }\end{array}$ & Manual & $\begin{array}{l}\text { Especifica erros } \\
\text { típicos de } \\
\text { Omissão/Comissão }\end{array}$ \\
\hline SLIM/MAUD & \multicolumn{2}{|c|}{$\begin{array}{c}\text { Os analistas podem definir o } \\
\text { domínio a ser coberto, lista } \\
\text { de sugestões cobre } \\
\text { ergonômica, cognitiva e } \\
\text { organizacional } \\
\end{array}$} & $\begin{array}{l}\text { Números } \\
\text { produzidos pelo } \\
\text { analista }\end{array}$ & Manual & $\begin{array}{l}\text { Nenhum } \\
\text { especificado }\end{array}$ \\
\hline ATHEANA & \multicolumn{2}{|c|}{$\begin{array}{l}\text { Ergonômica, cognitiva e } \\
\text { organizacional }\end{array}$} & $\begin{array}{l}\text { Números } \\
\text { produzidos pelo } \\
\text { analista }\end{array}$ & Manual & $\begin{array}{c}\text { Opinião } \\
\text { especializada }\end{array}$ \\
\hline CREAM & \multicolumn{2}{|c|}{$\begin{array}{l}\text { Ergonômica, cognitiva e } \\
\text { organizacional }\end{array}$} & $\begin{array}{c}\text { Números } \\
\text { fornecidos pelo } \\
\text { método }\end{array}$ & Manual & 13 modos de erro \\
\hline MERMOS & \multicolumn{2}{|c|}{$\begin{array}{c}\text { Ergonômica, cognitiva e } \\
\text { organizacional }\end{array}$} & $\begin{array}{l}\text { Números } \\
\text { fornecidos pelo } \\
\text { método }\end{array}$ & Software & $\begin{array}{c}\text { Opinião } \\
\text { especializada com } \\
\text { auxílio de simulador }\end{array}$ \\
\hline
\end{tabular}

Revista Produção Online. Florianópolis, SC, v. 20, n. 4, p. 1170-1189, 2020. 
Quadro 1 - Comparativo entre métodos de HRA

(conclusão)

\begin{tabular}{|c|c|c|c|c|c|}
\hline \\
\hline \multirow[b]{2}{*}{ Técnica } & \multicolumn{2}{|c|}{ Tratamento específico } & \multirow[b]{2}{*}{$\begin{array}{l}\text { Estimação de } \\
\text { limites de } \\
\text { incerteza }\end{array}$} & \multirow[b]{2}{*}{$\begin{array}{l}\text { Identificação } \\
\text { do erro: } \\
\text { Reprodutibili } \\
\text { dade }\end{array}$} & \multirow[b]{2}{*}{$\begin{array}{l}\text { Identificação do } \\
\text { erro: Validação }\end{array}$} \\
\hline & $\begin{array}{l}\text { para Tarefa/ } \\
\text { Dependências } \\
\text { de erro }\end{array}$ & \begin{tabular}{|c|} 
para \\
Recuperação \\
(inclui ações \\
com \\
feedback)
\end{tabular} & & & \\
\hline THERP & $\mathrm{x}$ & $x$ & $\mathrm{x}$ & $\begin{array}{l}\text { Não faz } \\
\text { identificação } \\
\text { de erro }\end{array}$ & $\begin{array}{c}\text { Não faz } \\
\text { identificação de } \\
\text { erro }\end{array}$ \\
\hline SLIM/MAUD & & & & $\begin{array}{l}\text { Não faz } \\
\text { identificação } \\
\text { de erro }\end{array}$ & $\begin{array}{c}\text { Não faz } \\
\text { identificação de } \\
\text { erro } \\
\end{array}$ \\
\hline ATHEANA & $x$ & $x$ & $x$ & Média & Nenhum \\
\hline CREAM & & & $x$ & $\begin{array}{l}\text { Não faz } \\
\text { identificação } \\
\text { de erro }\end{array}$ & $\begin{array}{c}\text { Não faz } \\
\text { identificação de } \\
\text { erro }\end{array}$ \\
\hline MERMOS & $x$ & $x$ & $x$ & Média & Nenhum \\
\hline \multirow[b]{2}{*}{ Técnica } & \multicolumn{3}{|c|}{ Estimação HEP } & \multirow{2}{*}{\multicolumn{2}{|c|}{ Base de experiência }} \\
\hline & Validação & $\begin{array}{c}\text { Reprodutibili } \\
\text { dade }\end{array}$ & Sensibilidade & & \\
\hline THERP & $\begin{array}{l}1 \text { validação } \\
\text { conhecida; } \\
\text { amplamente } \\
\text { referido }\end{array}$ & Média & Baixa & \multicolumn{2}{|c|}{$\begin{array}{l}\text { Amplamente utilizada e } \\
\text { referenciada (nuclear, perfuração } \\
\text { de petróleo offshore e NASA) }\end{array}$} \\
\hline SLIM/MAUD & Nenhuma & Baixa & $\begin{array}{l}\text { Sensível em } \\
\text { uma cauda de } \\
\text { Prob. Dist. }\end{array}$ & \multicolumn{2}{|c|}{$\begin{array}{l}\text { Muitas aplicações nucleares dos } \\
\text { EUA }\end{array}$} \\
\hline ATHEANA & Nenhuma & Baixa & Média & \multicolumn{2}{|c|}{$\begin{array}{l}\text { U.S. nuclear, desmilitarização de } \\
\text { armas químicas nos EUA e } \\
\text { ferrovias }\end{array}$} \\
\hline CREAM & Nenhuma & Alta & Baixa & \multicolumn{2}{|c|}{$\begin{array}{l}\text { Amplamente utilizada (nuclear, } \\
\text { perfuração de petróleo offshore e } \\
\text { aplicações da NASA) }\end{array}$} \\
\hline MERMOS & Nenhuma & Média & Alta & \multicolumn{2}{|c|}{$\begin{array}{l}\text { Planta energia nuclear da } \\
\text { Électricité de France }\end{array}$} \\
\hline
\end{tabular}

Fonte: Adaptado pelos autores baseados em Chandler (2006)

No caso desse trabalho, quatro das cinco técnicas escolhidas de avaliação da confiabilidade humana são modeladas em relação ao contexto e apenas a THERP segue modelo relacionado as tarefas. Outra atribuição válida é o nível de esforço necessário para calcular HEPs, enquanto, as técnicas THERP e CREAM requerem um esforço médio, sendo necessária até duas semanas, os métodos ATHEANA, SLIM/MAUD e MERMOS requerem alto esforço, significando mais de duas semanas incluindo auxílio de especialistas, recursos e tempo mais intensos.

Como mencionado antes, uma ampla gama de variados modelos ou técnicas de HRA estão disponíveis, cada um com suas próprias características. No entanto, 
ter alguns atributos identificáveis permite examinar e discutir a base do modelo de maneira construtiva.

\section{CONSIDERAÇÕES FINAIS}

O artigo atendeu a proposta de revisar bibliograficamente cinco métodos de avaliação da confiabilidade humana, sendo estes métodos relevantes tanto em indústrias que tem o fator humano como o elo crítico do sistema quanto na área acadêmica, em que foi constatado como um tema ainda incipiente no Brasil.

O método THERP preza pelo cálculo da probabilidade de desempenho bemsucedido das atividades necessárias para a realização de uma missão. Utiliza modelagem representacional de ações humanas (árvores de evento de HRA) e estimativa de HEPs (os cálculos baseiam-se em taxas de erro pré-definidas, onde o sucesso é definido como o seu complemento), através da predição das taxas de falha humana pode analisar a degradação do sistema homem-máquina.

Em relação ao método SLIM-MAUD, o objetivo é estimar as HEPs por meio de um procedimento interativo baseado em computador, considerando as probabilidades relativas de diferentes erros em função de vários fatores que podem afetar o desempenho humano. O resultado pode ser usado em árvores de eventos e falhas de HRA.

Já no método ATHEANA, o objetivo é identificar situações que causariam ações inseguras do operador (contextos forçando erros). Em princípio, estes devem abranger fatores cognitivos, organizacionais e ergonômicos. Além de possuir capacidade de realizar análise preditiva de tarefas (ou identificação de erros) e análise retrospectiva de eventos, promete determinar vulnerabilidades cognitivas em equipes, que podem não ser descobertas ao aplicar outros métodos de HRA

Quanto ao método CREAM, este preconiza identificar tarefas que demandam cognição humana, para determinar as condições que reduzem suas confiabilidades, e quais ações podem se tornar uma fonte de risco; e assim desenvolver e especificar modificações para melhorar estas condições. Para isso, entre outros é preciso realizar uma classificação de desempenho humano com foco em modos de erro e consequências (fenótipos) e causas (genótipos). 
O método MERMOS prevê a probabilidade de as equipes causarem erros. $\mathrm{O}$ método preconiza identificar os estados observando as equipes em operação (respondendo a acidentes no simulador), considerando os registros anteriores e as considerações de especialistas. Por isso, é centrado no uso de informações do simulador e dados coletados por observadores denotando as ações da equipe quando eles estão respondendo a um acidente simulado.

Os procedimentos de predição e análise de falhas, até então voltados para projetos e processos industriais, associados a falhas de equipamentos, podem ser ampliados para utilização no controle e prevenção das falhas cometidas por pessoas. Conclui-se que os objetivos propostos nesta pesquisa referentes a compreensão da utilidade de determinados métodos de avaliação de confiabilidade humana, evidenciando-se as semelhanças e, principalmente, as diferenças entre os métodos abordados, foram cumpridos. A pesquisa realizada contribui para o estudo da confiabilidade humana, cujo tema é pouco abordado na literatura científica apesar de sua relevância e aplicabilidade em contextos nacional e internacional.

\section{REFERÊNCIAS}

ALVARENGA, M. A. B; FONSECA, R. A. Comparison of the therp quantitative tables with the human reliability analysis techniques of second generation. In: INTERNATIONAL NUCLEAR ATLANTIC CONFERENCE (INAC), Rio de Janeiro. [Anais eletrônicos...]. Rio de Janeiro, 2009. Disponível em:

http://www.iaea.org/inis/collection/NCLCollectionStore/ Public/40/103/40103869.pdf. Acesso em: 02 de mar. 2019.

BORING, R. L. Top-down and bottom-up definitions of human failure events in human reliability analysis. In: HUMAN FACTORS AND ERGONOMICS SOCIETY ANNUAL MEETING, 58., 2014. [Proceedings...]. Los Angeles: Sage Publications, 2014. p. 563-567. https://doi.org/10.1177/1541931214581119

BYE, A; BRAARUD, P. Ø. Improving the empirical basis for human reliability assessment by simulator experiments. In: INTERNATIONAL CONFERENCE ON PROBABILISTIC SAFETY ASSESSMENT AND MANAGEMENT,. 7., 2004. [Proceedings...]. Berlin, 2004. https://doi.org/10.1007/978-0-85729-410-4 196

CHANDLER, F. T; CHANG, J. Y. H; MOSLEH, A; MARBLE, J. L; BORING, R. L; GERTMAN, D. I. Human reliability analysis methods selection guidance for nasa. Technical Report of National Aeronautics and Space Administration (NASA) / Office of Safety and Mission Assurance (OSMA), Washington, 2006. 
DEKKER, S. W. A. The field guide to understanding human error. Aldershot: Ashgate Publishing Co., 2006.

GERTMAN, D. I; BLACKMAN, H; MARBLE, J; BYERS, J; SMITH C. The spar-h human reliability analysis method. U.S. Nuclear Regulatory Commission (NUREG) - Idaho National Laboratory, Washington, 2005.

HEALTH AND SAFETY EXECUTIVE. Understanding human failure. Disponível em: http://www.hse.gov.uk/construction/lwit/assets/downloads/human-failure.pdf. Acesso em: 09 de fev. 2019.

HOLLNAGEL, E. Cognitive reliability and error analysis method. 1. ed. New York: Elsevier, 1998. https://doi.org/10.1016/B978-008042848-2/50001-4

KUMAMOTO, H; HENLEY, E. J. Probabilistic risk assessment and management for engineers and scientists. 2 ed. New York: IEEE Press, 1996.

KOMNINAKIS, D; PIRATELLI, C. L; ACHCAR, J. A. Análise de Confiabilidade para Formulação de Estratégia de Manutenção de Equipamentos em uma Empresa da Indústria Alimentícia. Revista Produção Online, Florianópolis, SC, v. 18, n. 2, p. 560-592, 2018. https://doi.org/10.14488/1676-1901.v18i2.2871

LAFRAIA, J. R. B. Manual de confiabilidade, mantenabilidade e disponibilidade. 1. ed. Rio de Janeiro: Qualitymark, 2001.

NASCIMENTO NETO, M. P. Proposição de uma sistemática para avaliação de confiabilidade humana em mina a céu aberto. Tese (Doutorado em Engenharia) Programa de Pós-Graduação em Engenharia de Produção, Universidade Federal do Rio Grande do Sul, Porto Alegre, 2014. Disponível em:

https://www.lume.ufrgs.br/bitstream/handle/10183/127866/000971494.pdf?sequence=1. Acesso em: 12 mar. 2019. https://doi.org/10.22456/2317-8558.50228

ØWRE, F. The history of HAMMLAB. In: SKJERVE, A. B. ; BYE, A. (eds.). Simulator-based human factors studies across 25 years. London: Springer-Verlag, p.13-44. 2011. https://doi.org/10.1007/978-0-85729-003-8 2

PALLEROSI, C. A; MAZZOLINI, B. P. M; MAZZOLINI, L. R. Confiabilidade humana: conceitos, análises, avaliação e desafios. São Paulo: All Print Editora, 2011.

PESME, H; LE BOT, P; MEYER, P. A Practical Approach of the MERMOS Method, Little Stories to Explain Human Reliability Assessment. In: HUMAN FACTORS AND POWER PLANTS (HPRCT) AND INSTITUTE OF ELECTRICAL AND ELECTRONICS ENGINEERS (IEEE) ANNUAL MEETING, 13., 2007. [Proceedings...]. Monterey, 2007. https://doi.org/10.1109/HFPP.2007.4413220

PINTO, J. M. O. Análise de falhas humanas em um sistema digital de uma usina nuclear utilizando uma metodologia híbrida DFM/ATHEANA/FUZZY. Tese (Doutorado em Engenharia) - Programa de Engenharia Nuclear, Instituto Alberto Luiz Coimbra de PósGraduação e Pesquisa de Engenharia (COPPE), Universidade Federal do Rio de Janeiro (UFRJ), Rio de Janeiro, 2014. Disponível em: http://antigo.nuclear.ufri.br/DScTeses/teses2014/Tese Jonathan Marcello.pdf. Acesso em: em: 16 mar. 2019. 
PRODANOV, C. C; FREITAS, E. C. F. Metodologia do trabalho científico: métodos e técnicas da pesquisa e do trabalho acadêmico. 2. ed. Novo Hamburgo: Feevale, 2013.

REASON, J. Understanding adverse events: human factors. Manchester: BMJ Quality \& Safety, v. 4, n. 2, p. 80-89,1995. https://doi.org/10.1136/qshc.4.2.80

RIGBY, L. V. The nature of human error. In: ANNUAL TECHNICAL CONFERENCE TRANSACTIONS OF THE AMERICAN SOCIETY FOR QUALITY CONTROL.

[Proceedings...]. Milwaukee, p. 457-466, 1970.

SERRANO, E. J. M. Conceito, classificação e quantificação da fiabilidade humana na relação homem-máquina. Dissertação (Mestre em Engenharia Mecânica) - Departamento de Engenharia Mecânica (DEM), Instituto Superior de Engenharia de Lisboa (ISEL), Lisboa, 2009. Disponível em: https://repositorio.ipl.pt/bitstream/10400.21/434/1/Dissertação.pdf.

Acesso em: 20 mar. 2019.

SILVA, B. G. J; LISBOA, A. P; SANTOS, A. H. A; SANTANA, K. S; SILVA, I. P. Confiabilidade Humana: uma abordagem atual do erro humano. In: SIMPÓSIO DE ENGENHARIA DE PRODUÇÃO DE SERGIPE (SIMPROD), 9., 2017. São Cristóvão. [Anais eletrônicos...]. Sergipe, 2017. Disponível em:

https://ri.ufs.br/bitstream/riufs/7676/2/ConfiabilidadeHumanaAbordagemErro.pdf. Acesso em: 21 mar. 2018.

SPURGIN, A. J. Human reliability assessment: theory and practice. Boca Raton: CRC, 2010. https://doi.org/10.1201/9781420068528

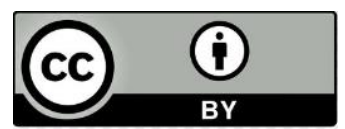

Artigo recebido em: 25/04/2019 e aceito para publicação em: 20/09/2020 DOI: http://dx.doi.org/10.14488/1676-1901. v20i4.3629 